\title{
Self-propulsion of droplets via light-stimuli rapid control of their surface
}

\section{tension}

Anna Yucknovsky, ${ }^{\mathrm{a}}$ Benjamin B. Rich, ${ }^{\mathrm{b}}$ Ariel Westfried, ${ }^{\mathrm{a}}$ Boaz Pokroy, ${ }^{\mathrm{b}}$ and Nadav Amdursky ${ }^{\mathrm{a}, *}$

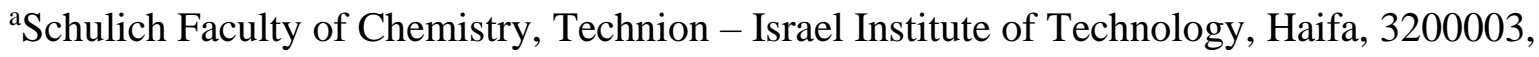

Israel; ${ }^{b}$ Department of Materials Science \& Engineering, Technion-Israel Institute of

Technology, Haifa, 3200003 Israel

*Corresponding author, e-mail: amdursky@technion.ac.il

Keywords: Droplets; Photoacids; Photobases; Marangoni effect

\begin{abstract}
Biology demonstrates many examples of response and adaptation to external stimuli, and here we focus on self-propulsion (motion) while presenting several self-propelling droplet systems responsive to $\mathrm{pH}$ gradients. We use light as the gating source to gain reversibility, avoid the formation of chemical wastes, and control the self-propulsion remotely. To achieve light-stimuli ultra-fast response, we use photoacids and photobases, capable of donating or capturing a proton, respectively, in their excited-state. We control the movement and directionality of the droplet's self-propulsion by introducing the photo-acid/base either in bulk solution, on the surface of the droplet, or inside the droplet. We show that proton transfer between the photo-acid/base and the droplet results in a rapid change in the droplet's surface tension, which induces the selfpropulsion movement. The high versatility of our systems together with a record-breaking ultrafast response to light makes them highly attractive for the design of various controlled cargocarrier systems.
\end{abstract}




\section{Introduction}

Motion is an inherent basic functionality in our existence, manifested at all levels; from our entire entity down to the single molecular and macromolecular levels, such as the movement of motor proteins, the self-assembly of (bio-)molecules to higher hierarchical structures, and much more. A crucial part of motion is its control, meaning when and how to initiate the process, controlling the direction and magnitude of the movement, and most importantly, when and how to stop it. Inspired by nature, various stimuli-responsive systems and materials have been developed using (macro-)molecular motion for the making of diverse functionalities ${ }^{1-5}$. One of the heavily studied stimuli-responsive systems is surfactant-/lipid-based droplets, in which several stimuli-responsive motions, i.e., self-propulsion, of droplets have been designed, using various modes of stimuli, such as: chemical, temperature, and light stimuli. ${ }^{6-17}$ Such systems can mimic the motion of living cells as similar stimuli are present in nature, and can also result in our fundamental understanding of early origin of life events.

The movement of droplets is linked with non-uniform surface tension (ST), resulting in fluid flow, known as the Marangoni effect ${ }^{18-19}$. This effect is based on an asymmetric exposure of the droplet surface to a chemical cue, i.e., chemotaxis, whereas ion or $\mathrm{pH}$ gradients are most common $^{20-22}$. The asymmetry in the droplet/solution interface creates a non-uniform change in the ST. Light is a convenient tool to control the asymmetry and ST of droplets, and accordingly the motion of droplets. To use light as stimuli, there is a need of photochromic molecules next to the droplets, undergoing a photochemical process. To date, the commonly used families of photochromic molecules for mediating droplet motion are azobenzenes that undergo trans-cis photoisomerization $^{23}$, and spiropyrans that undergo reversible photocleavage resulting in a release of a proton ${ }^{24-26}$. Nonetheless, a yet-to-be-resolved challenge in the use of such photochromic molecules is the associated timescales and especially the reversibility of the dynamic process. 
Herein, we propose a new approach to chemophototaxis for gaining fast sub-second responses not only to turning on the light, but also for the reversible process of turning the light off, which is based on the use of Brønsted-Lowry photoacids and photobases. This class of organic molecules undergoes a dramatic change in their dissociation equilibrium constant (pKa) upon light excitation $^{27}$. Thus, only in their electronically excited state do photoacids and photobases behave as strong acids and bases. The above-mentioned spiropyrans are also referred to as photoacids since their photocleavage process from spiropyran to merocyanine involves the release of a proton, however, there is a large difference in terms of mechanism and timescales relative to Brønsted-Lowry photoacids. In this context, we hypothesize that the fast excited-state proton transfer (ESPT) of Brønsted-Lowry photoacids and photobases can induce rapid chemical changes on the surface of $\mathrm{pH}$-sensitive droplets, and initiate their motion. We show here a variety of different droplet systems involving the use of photoacids and photobases either within the droplet, on its surface, or in solution, whereas we use light to self-propel and to guide the movement of the droplet.

\section{Results}

The systems that we have developed here contain a nitrobenzene oil droplet (colored red for ease of observation) in an aqueous phase, a surfactant, a photoacid or photobase, and a light source. Using these components, we created five configurations that differ in the location of the photoacid or photobase, their concentration, droplet structure, type of surfactant, and mode of irradiation (Figure 1). In the first two systems, we added the photoacid or photobase to the aqueous solution (Figure 1a and 1b). In the third system, we added a different type of photoacid, which is soluble only in the organic phase, meaning that it was incorporated into the droplet itself (Figure 1c). In the fourth system, we introduced a photoacid by creating a complex two-layer drop, in which the outer organic phase envelops the inner photoacid-containing aqueous phase

(Figure 1d). Our main experimental hypothesis toward moving the droplet by light is based on a proton transfer event between the surfactant and the photoacid or photobase, as well as on the 
electrostatic interactions between them. The driving force for these processes is the irradiation of the system with nonuniform light intensity for this we used light dispersion from a LED to create a light gradient, the direction of which defined the droplet's direction of movement. However, in our last fifth system we illuminated the drop with a focused laser beam, allowing precise navigation of the droplet in various directions (Figure 1e).

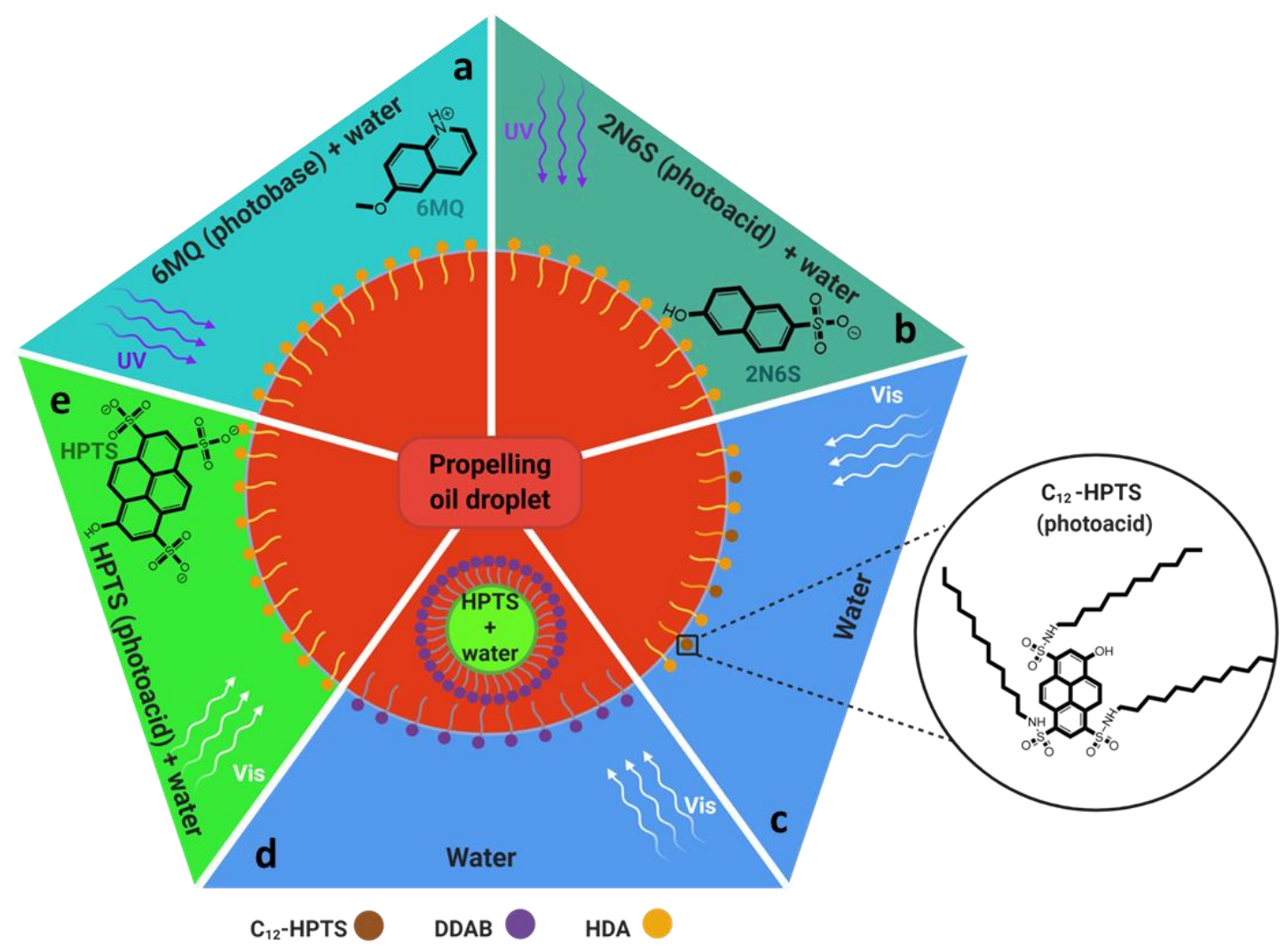

Figure 1. Schematic presenting the various systems of propelling an oil droplet. (a), (b)

Droplet in photobase and photoacid solution, respectively, propelled using UV light irradiation.

(c) Droplet containing oil-soluble photoacid in aqueous solution, propelled using Vis light irradiation. (d) Complex w/o droplet containing photoacid in its inner aqueous layer, propelled using Vis light irradiation. (e) Droplet in photoacid solution, propelled using Vis light focused laser beam irradiation. 


\section{Oil droplet in a photobase solution}

Our first system consists of an oil droplet within an aqueous solution containing the 6methoxyquinoline (6MQ) photobase. 6MQ has pKa values of 5.2 and 11.8 in its ground and excited state respectively, ${ }^{28}$ hence it only protonates with light irradiation (Figure S1a). As shown in Figure 2a and Movie S1, the drop was illuminated from a $340 \mathrm{~nm}$ LED placed above the sample. Due to the dispersion of the beams, a light intensity gradient is developed in the petri dish (further details in the experimental section), resulting in a nonuniform 6MQ excitation, and subsequently, a pH gradient. The nitrobenzene droplet in this section contained 2-hexyldecanoic acid (HDA) (in a 1:1 ratio), which acts as a pH-sensitive carboxylic acid surfactant. The working principle of this section was to induce the droplet self-propulsion following an ESPT process from HDA to 6MQ (Figure 2a). To allow this, the initial protonation states of both 6MQ and HDA are important. Accordingly, the solution $\mathrm{pH}$ was adjusted to $\mathrm{pH} 6$, so that the 6MQ would be deprotonated, and the HDA (having a pKa of 7) is protonated. ${ }^{26,29-30}$ Upon illumination with the LED, we noticed the self-propulsion of the droplet away from the light with an average velocity of $0.2 \pm 0.09 \mathrm{~m} / \mathrm{s}$, calculated from repeated measurements (such as the one in Figure $\mathbf{2 b}$, showing the trajectory in a typical experiment).

To reveal the mechanism of the light-triggered self-propulsion of the droplet, we turned to optical tensiometer measurements and monitored the ST fluctuations upon light irradiation in the same droplet-solution system (Figure 2c). The figure presents multiple $10 \mathrm{sec}$ light on/off cycles, during which ST drops and rises. The light-triggered drop in ST is due to the HDA deprotonation following the ESPT process from HDA to 6MQ. The high repeatability of the process is due to the reversibility of the 6MQ, which captures protons in its electronically excited state, and releases them in its ground state. The fast proton capture and release of 6MQ results in a very fast change in the droplet's ST, with a calculated response time of $32 \mathrm{~ms}$ for the light-triggered drop in ST, and $32 \mathrm{~ms}$ for the reversible process upon turning off the light (See Figure S2a for a highresolution scan of the ST). We further monitored the change in ST upon irradiating with different 
light intensities (Figure 2d). As shown in the figure, the larger the intensity of the light, the greater the drop in ST. Importantly, the self-propulsion and the corresponded change in ST were only due to the presence of the excited photobase, whereas controls of using light without the presence of the photobase or before turning on the light did not result in any self-propulsion or a change in ST (Figure S3).
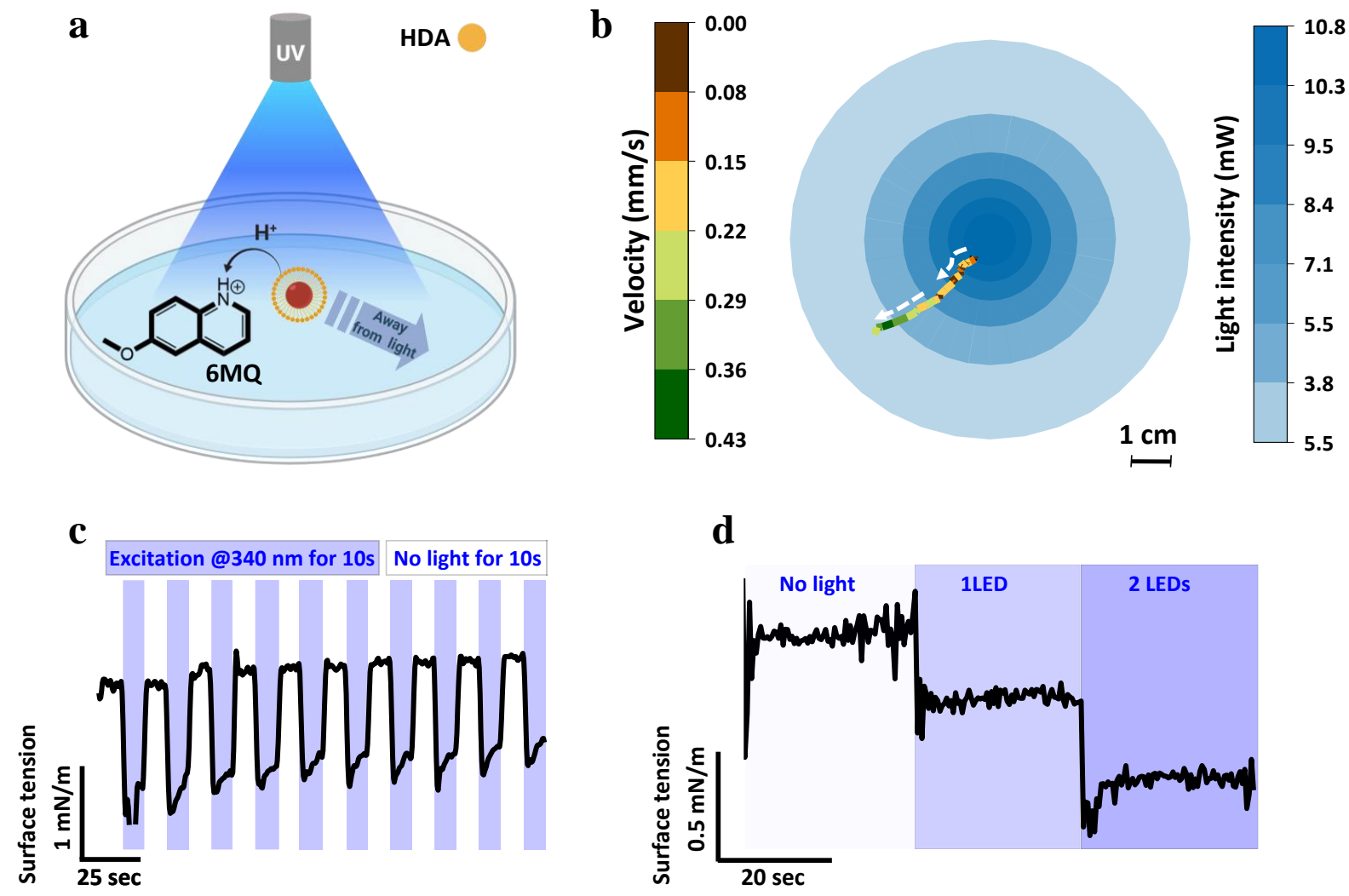

Figure 2. Oil droplet in a photobase solution. (a) A schematic representation of the experimental design. (b) The trajectory of an oil droplet showing the light-triggered selfpropulsion along with its velocity, in accordance with the light gradient in the petri dish. (c) Light-triggered changes in ST following cycles of $10 \mathrm{sec}$ illumination and darkness. (d) Lightintensity-dependent change in ST upon illuminating with one or two LED's. $\lambda_{\mathrm{ex}}=340 \mathrm{~nm}$ for all panels.

\section{Oil droplet in a photoacid solution}

The setup in this section is rather similar to the previous one, albeit changing the solution from a photobase containing solution to a photoacid containing one. The photoacid that we chose to use 
here is 2-naphthol-6-sulfonate (2N6S) with pKa values of 9.5 and 1.7 in its ground and excited state, respectively ${ }^{31}$ (Figure S1b). Our working principle in this section (Figure 2a) is to enable light-triggered ESPT from 2N6S to HDA, resulting in a change in ST and the self-propulsion of the droplet away from the light. To enable this, we have adjusted the $\mathrm{pH}$ in the solution to $\sim 8$, hence 2N6S is protonated, and HDA is deprotonated. We have followed the trajectory of the droplet upon illumination (Figure 3b and Movie S2), and we indeed observed that the droplet propelled away from the light with an average velocity of $0.12 \pm 0.05 \mathrm{~m} / \mathrm{s}$, which is only due to the presence of the excited photoacid (Figure S3). Interestingly, we observed a similar direction to the droplet's propulsion while using both the 6MQ photobase as well as the 2N6S photoacid. Additionally, the change in measured ST upon multiple light/dark cycles (Figure 3c) shows a similar trend, in which we observed a sharp drop in ST upon turning the light on and a sharp rise in ST upon turning the light off (see below for a detailed discussion on the differences between the systems). Similar to the 6MQ system, the reversibility and fast kinetics of the system is due to the photoprotolytic cycle of 2N6S, undergoing proton dissociation in its excited-state and proton recombination upon returning to its ground state. The change in ST in the 2N6S system was faster than the instrument time resolution, which is $16 \mathrm{~ms}$ (See Figure S2b for a high-resolution scan of the ST). Hence, in the 2N6S system, the light-triggered drop in ST $(<16 \mathrm{~ms})$, as well as the reversible process upon turning off the light $(<16 \mathrm{~ms})$ are faster than the ones calculated for the 6MQ photobase system. This is probably due to the stronger photoacidity of 2N6S compared to the photobasisity of 6MQ, measured in terms of the pKa gap $(\Delta \mathrm{pKa})$ between ground and excited state, with values of 7.8 and 6.6 for $2 \mathrm{~N} 6 \mathrm{~S}$ and 6MQ, respectively. In accordance with the previous system, we observed a light-intensity-dependent change in ST in this system as well (Figure 3d). 

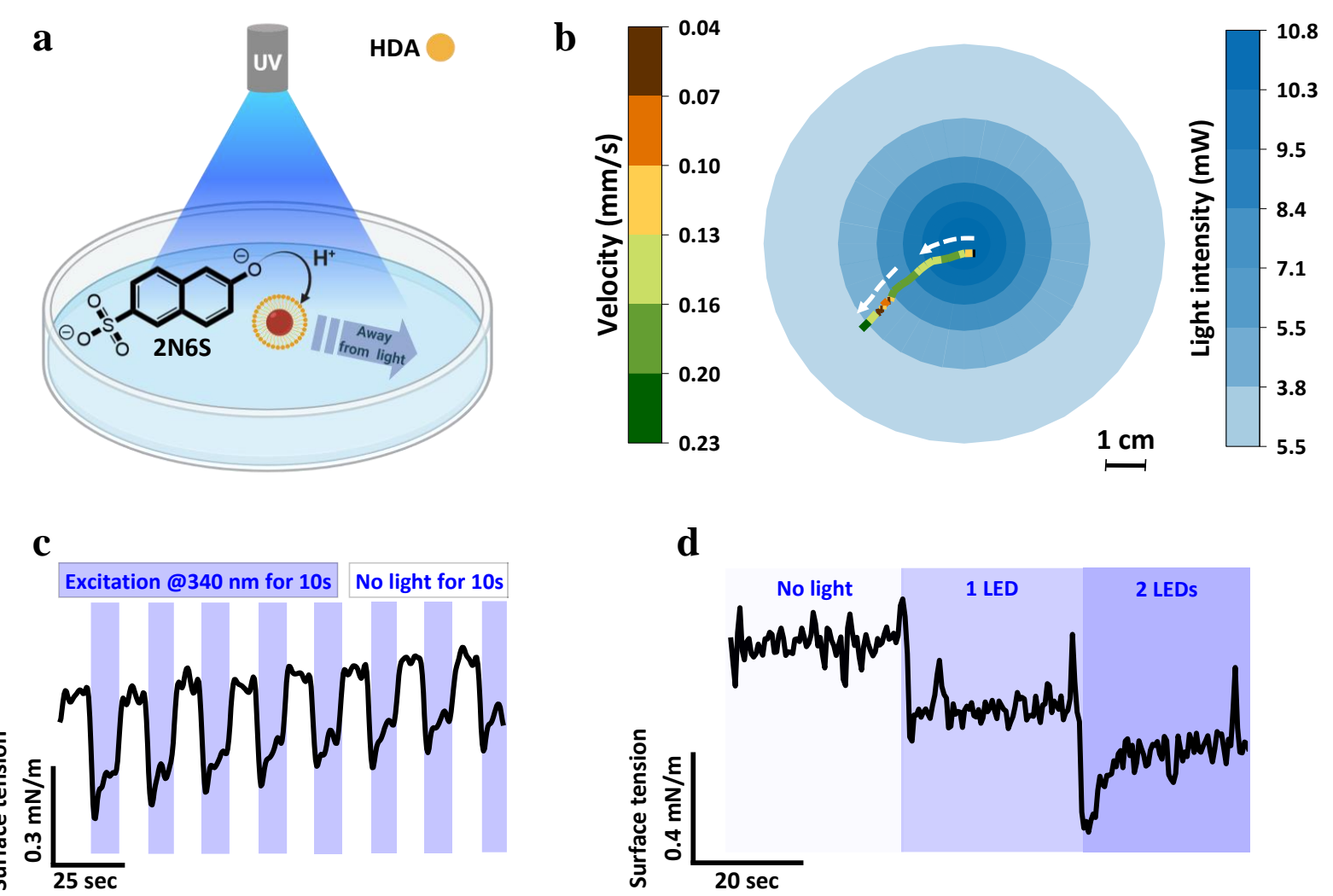

Figure 3. Oil droplet in a photoacid solution. (a) A schematic representation of the

experimental design. (b) The trajectory of an oil droplet showing the light-triggered self-

propulsion along with its velocity, in accordance with the light gradient in the petri dish. (c)

Light-triggered changes in ST following cycles of 10 sec illumination and darkness. (d) Lightintensity-dependent change in ST upon illuminating with one or two LED's. $\lambda_{\mathrm{ex}}=340 \mathrm{~nm}$ for all panels.

\section{Oil droplet with a tethered photoacid}

While in the systems discussed above we have used solvated photobase or photoacid, in this system we tethered the photoacid directly onto the surface of the droplet. To do so, we have used our recently developed photoacid composed of an HPTS where three long 12 carbon alkyl chains have been attached to its sulfonates ( $\mathrm{C}_{12}$-HPTS) (Figure S1c) ${ }^{32}$ As in our second system, we have used $\mathrm{HDA}$ as a proton acceptor and maintained initial $\mathrm{pH}$ values to have a protonated $\mathrm{C}_{12^{-}}$ HPTS and a deprotonated HDA. Accordingly, our working principle here (Figure 4a) is a lighttriggered ESPT from the tethered $\mathrm{C}_{12}$-HPTS photoacid to the HDA, resulting in a considerable 
change in ST and the self-propulsion of the droplet. The first important observation is the opposite directionality of the light-triggered self-propulsion of the droplets which moved toward the light, instead of away from the light as was seen in all the previous systems, with a measured average velocity of $0.05 \pm 0.03 \mathrm{~m} / \mathrm{s}$ (See Figure $\mathbf{4 b}$ and Movie S4 for a typical trajectory, and as in the previous systems, the movement was only due to the presence of the excited tethered photoacid, see Figure S4). In line with the reversed direction of the droplet's movement, also the ST measurements here show a reversed trend, exhibiting a light-triggered sharp increase in ST, followed by a sharp drop upon turning the light off (Figure 4c). Similar to the first two systems, also here the ST changes are fully reversible and can be repeated multiple times due to the reversible proton release and capture of $\mathrm{C}_{12}$-HPTS. An additional important observation shared among all of our first three systems is the correlation between the magnitude of the lighttriggered change in ST to the measured speed of the droplet upon illumination, whereas the larger the magnitude the faster the movement.
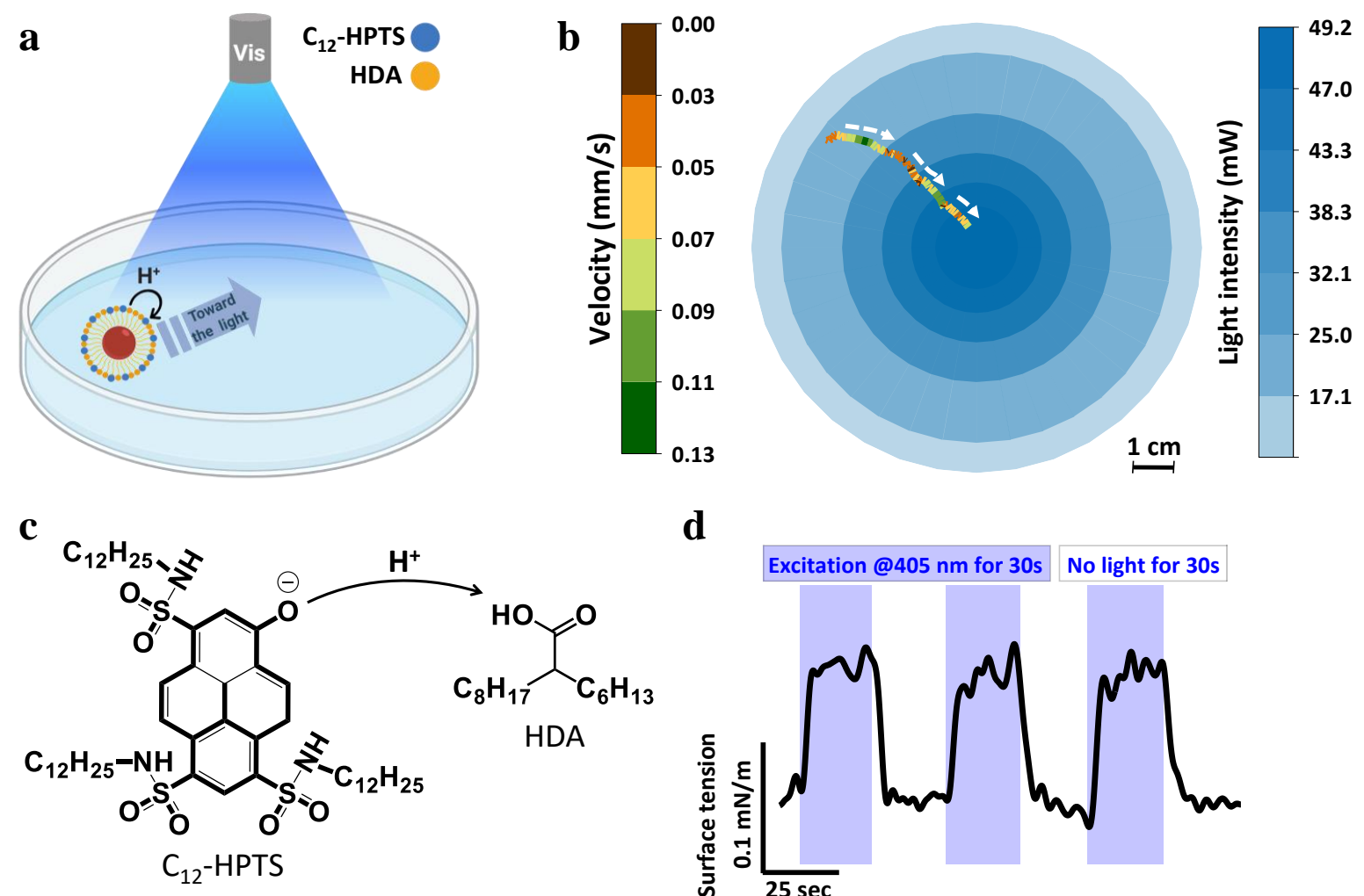

Figure 4. Oil droplet with a tethered photoacid. (a) A schematic representation of the experimental design (b) The trajectory of an oil droplet showing the light-triggered self- 
propulsion along with its velocity, in accordance with the light gradient in the petri dish. (c) Light-triggered changes in ST following cycles of $30 \mathrm{sec}$ illumination and darkness. $\lambda_{\mathrm{ex}}=405 \mathrm{~nm}$ for all panels.

\section{Water/oil/water system containing an inner photoacid phase}

Till now, we used 'simple' oil droplets. In this section, we designed a complex droplet with compartmentalization, in which an aqueous photoacid solution was encapsulated inside the oil layer. To do so, we have created a droplet from didecyldimethylammonium bromide (DDAB) in nitrobenzene (containing the oil red dye), to which an aqueous phase was injected containing the 8-hydroxypyrene-1,3,6-trisulfonic acid (HPTS) photoacid with pKa values of 7.4 and 0.4 in its ground and excited state, respectively ${ }^{33}$ (Figure S1d). The working principle here (Figure 5a) is that upon exciting the HPTS at $405 \mathrm{~nm}$, it will undergo proton dissociation, which in turn will change the ionic strength of the aqueous phase, thus affecting the ST between the aqueous and organic layers, leading eventually to the self-propulsion of the droplet.

As shown in the trajectory in Figure 5b and Movie S3, upon illumination with a $405 \mathrm{~nm}$ LED, the non-uniform light gradient causes the droplet's self-propulsion away from the light with an average velocity of $0.08 \pm 0.05 \mathrm{~m} / \mathrm{s}$ (which was only due to the encapsulated and excited photoacid, Figure S5). The ST measurements show a significant difference between the current system and the above-mentioned ones. While in the previous systems, we have followed the changes in the ST between the oil droplet and the bulk aqueous solution surrounding it, here the light-triggered photoprotolytic process takes place within the aqueous phase inside the oil droplet. Hence, the main ST that is being affected is the one between the internal aqueous and the organic layer of the droplet (though we cannot exclude subsequent changes in the ST between the bulk aqueous solution and the organic layer). As shown in Figure 5c, the light-triggered change in the ST of this system is showing a rather slow increase in the ST upon light triggering followed by a slow drop in ST after turning the light off. Even though the rise/drop cycles were repeatable, the 
ST did not reach its initial value after each cycle, which can be ascribed to HPTS degradation under continuous irradiation, meaning that not all of the released protons could recombine.

Interestingly, while both this system and the previous one showed a light-triggered increase in ST, this change has resulted in a different direction of the droplet's self-propulsion (which will be discussed below).
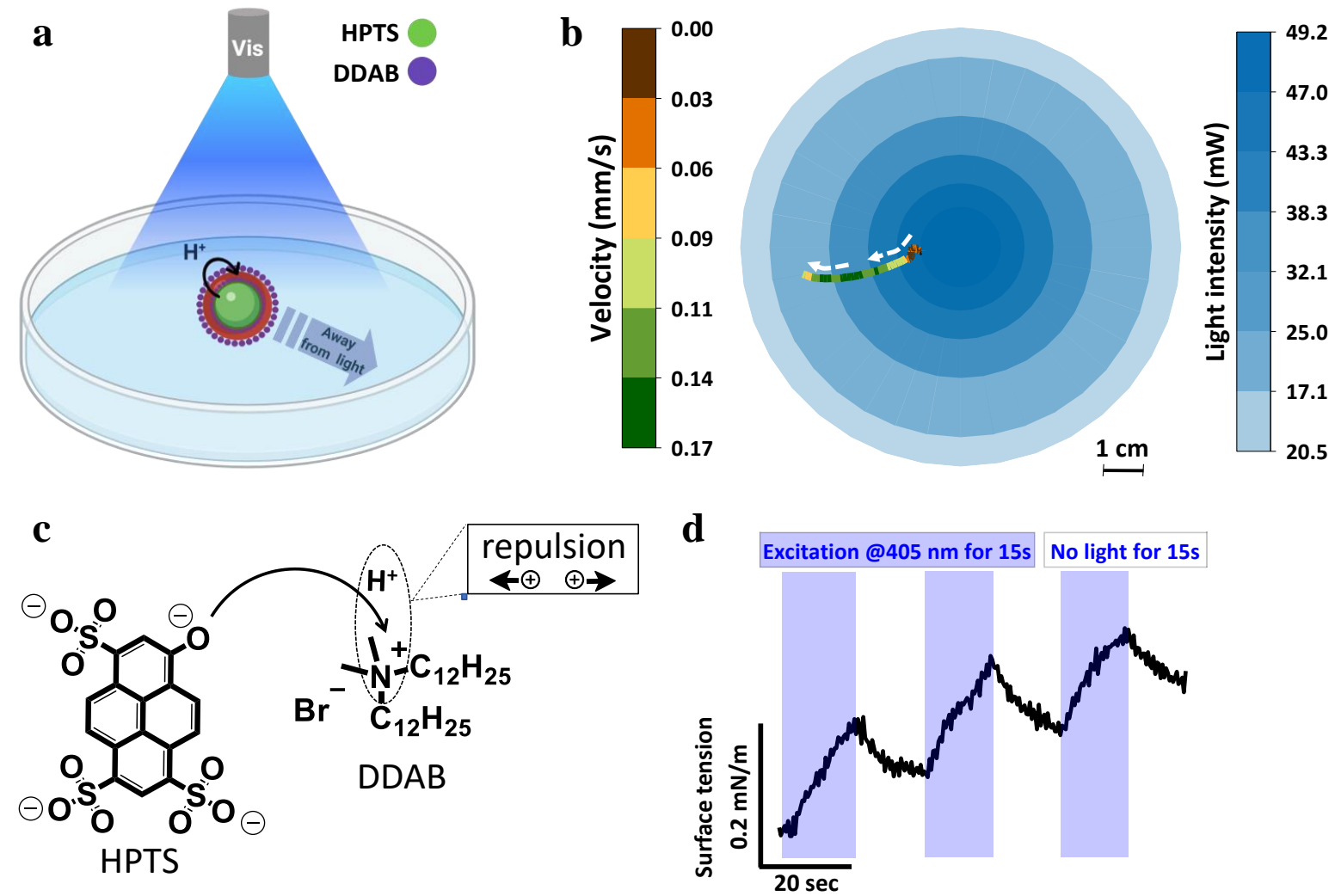

Figure 5. Complex oil droplet containing inner photoacid solution. (a) A schematic

representation of the experimental design (b) The trajectory of an oil droplet showing a lighttriggered self-propulsion along with its velocity, in accordance with the light gradient in the petri dish. (c) Light-triggered changes in ST following cycles of $15 \mathrm{sec}$ illumination and darkness. $\lambda_{\mathrm{ex}}=405 \mathrm{~nm}$ for all panels.

\section{Laser guided oil droplet in a photoacid solution}

In our last system, we introduced a different setup, in which the oil droplet was guided along a specific pathway using a laser pen, unlike the fixed LED position and accordingly light gradient in our previous systems. The experimental setup was similar to that of our second system, i.e., a 
nitrobenzene:HDA oil droplet in an aqueous solution containing a photoacid. However, here we used the HPTS photoacid and not the 2N6S one that was used above. The main reason for choosing the HPTS photoacid over any naphthol-based one, is the visible wavelength absorbance of HPTS (naphthols absorb only in the UV), allowing us to use a commercially available laser pen that emits at $405 \mathrm{~nm}$. The working principle here (Figure 6a) is identical to the second system, involving ESPT from the photoacid to HDA resulting in the self-propulsion of the droplet away from the light. Since here we are interested in the specific guidance of the droplet, we focused mainly on controlling the direction and the speed of the movement. To modulate the speed of the droplet, we have used different concentrations of HPTS in the solution. Higher concentrations of HPTS will result in more protons being released upon excitation causing a larger change in the ST, which, in turn, will eventually lead to faster droplet speeds (quantitative estimation of the extent of droplet protonation upon varying HPTS concentration can be found in the SI). As can be seen in Figures $6 \mathbf{b}$ (for a straight pathway) and Figure S6 (for a curved pathway), we could direct the droplet in specific pathways (the arrows in the figure represent the planned direction, as can be observed in Movies S5 and S6). From the summary of several measurements (such as the ones presented in Figures 6b), we could observe a linear correlation between the concentration of HPTS in the solution and the speed of the droplet (Figure 6c), thus proving that the excited photoacid is the driving force for the self-propulsion movement.

However, in contrast to all of our control experiments (Figure S3-S5), in here we observed some movement of the droplet even without the presence of the photoacid in the solution (Figure S7). This is a result of using a higher intensity laser in oppose to low-intensity LED's used in our previous systems, and it is also in line with a previous study showing that a high power laserinduced heat formation can result in the movement of a droplet away from the light. ${ }^{34}$ Nevertheless, the movement of the droplet without the presence of the photoacid in solution was significantly slower and very hard to control its directionality (Figure S7). 


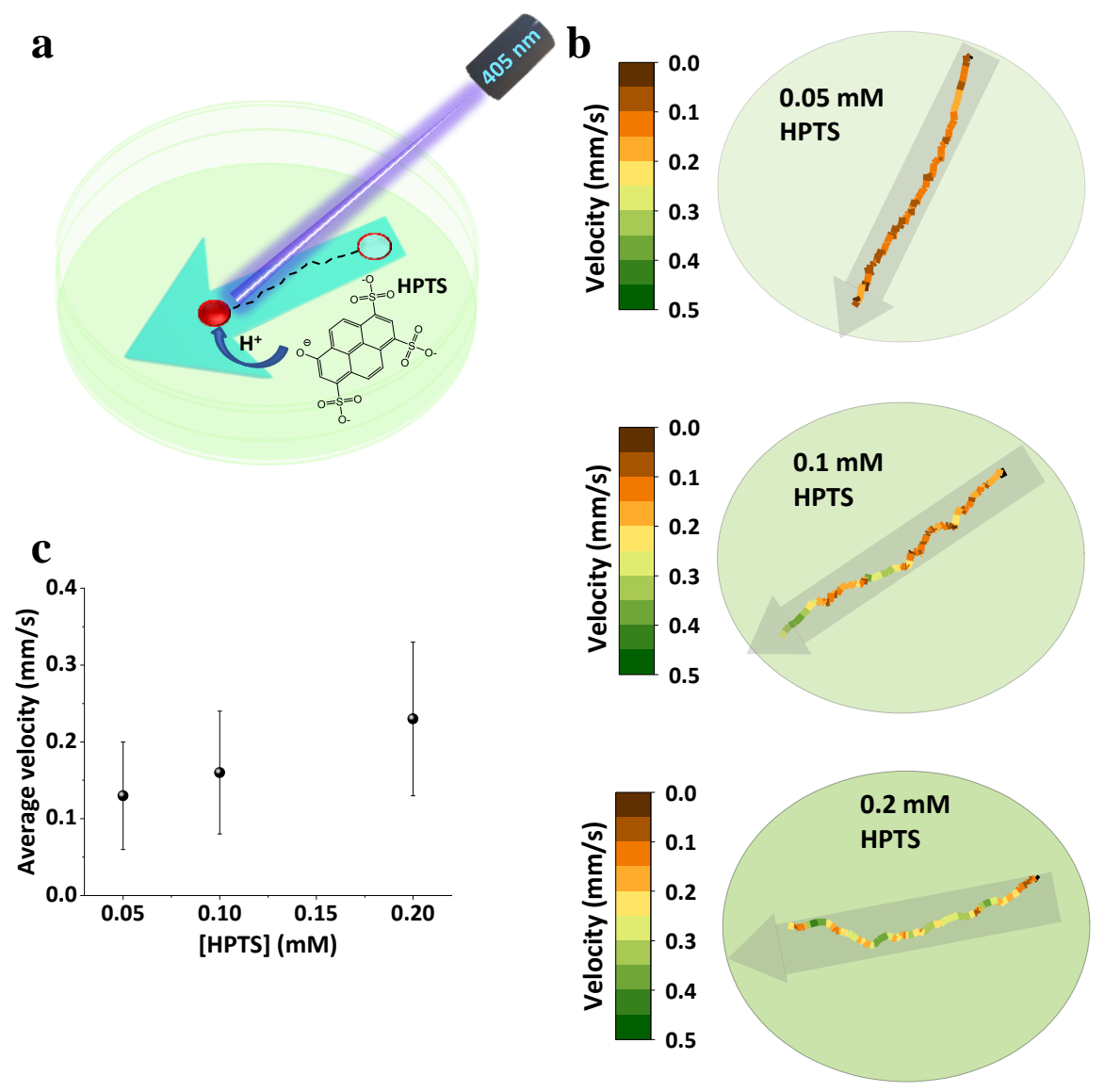

Figure 6. Laser guided oil droplet in a photoacid solution. (a) A schematic representation of the experimental design. (b) Trajectories of light-guided self-propelled oil droplets along with their velocities at $0.05,0.1$, and $0.2 \mathrm{mM}$ HPTS concentrations, respectively. (c) A summary of the averaged velocities of the droplets at different HPTS concentrations. $\lambda_{\mathrm{ex}}=405 \mathrm{~nm}$ for all panels.

\section{Discussion}

The role of ST in droplet self-propulsion mechanism: The key element of the self-propulsion mechanism is asymmetric ST along the droplet ${ }^{35}$. In our study, we created a ST gradient along the droplet/solution interface by non-uniform surfactant protonation or deprotonation of a photoacid or photobase, respectively. The ST gradient induces what is being referred to as Marangoni flows. Such flows are created when the surface stress needs to be balanced by the viscous stress, resulting in the formation of convection flows. These convective flows act opposite to the direction of the surface flows (Figure 7a). We can therefore explain all of our results in terms of this Marangoni effect (Figure 7). 
1) In the first system where the nitrobenzene:HDA droplet was in a photobase 6MQ solution

(Figure 7b), the light-triggering induces the proton capture by 6MQ and the deprotonation of HDA. The latter causes an HDA diffusion and convection along the droplet surface and the emergence of a tangential flow towards the light, while subsequent bulk flow causes droplet propulsion away from the light.

2) In the system where the nitrobenzene:HDA droplet was in a photoacid 2N6S solution (Figure 7c), the droplet surface is covered initially with deprotonated HDA (DA'), and upon lighttriggering the photoacid dissociates, resulting in $\mathrm{DA}^{-}$protonation. As before, a tangential flow towards the light emerges due to $\mathrm{DA}^{-}$diffusion and convection along the droplet surface, and subsequent bulk flow causes droplet propulsion away from the light. This mechanism is similar to the one operating in our last (fifth) system where the nitrobenzene:HDA droplet was in a photoacid HPTS solution.

3) In the system containing a nitrobenzene:HDA droplet with tethered $\mathrm{C}_{12}$-HPTS photoacid (Figure 7d), where both HDA and $\mathrm{C}_{12}$-HPTS are located on the droplet surface, the HDA is deprotonated ( $\left.\mathrm{DA}^{-}\right)$and $\mathrm{C}_{12}$-HPTS is protonated before light exposure. Upon light-triggering, the photoacid dissociates and $\mathrm{DA}^{-}$undergoes protonation, resulting in the emergence of tangential flow away from the light, due to both HDA and $\mathrm{C}_{12}$-HPTS${ }^{-}$diffusion and convection. This tangential flow is coupled to the convective flow, and eventually propels the droplet towards the light.

4) In the complex droplet configuration, the droplet is made up of a photoacid (HPTS) within a water core surrounded by an oil shell (Figure 7e). Before the irradiation, the interface between the layers is covered with the DDAB surfactant, but upon proton release within the aqueous core, electrostatic repulsion arises between the cationic surfactant and the protons, causing surfactant depletion, and subsequently liquid flow/propulsion. As a result, two types of convective flows are created (marked in red in Figure 7e): tangential flow in the aqueous core towards the light, and 
bulk flow in the oil shell away from the light. The resulting droplet movement has the same direction as the shell convective flows, most probably due to the larger mass of the oil shell compared to the core mass.
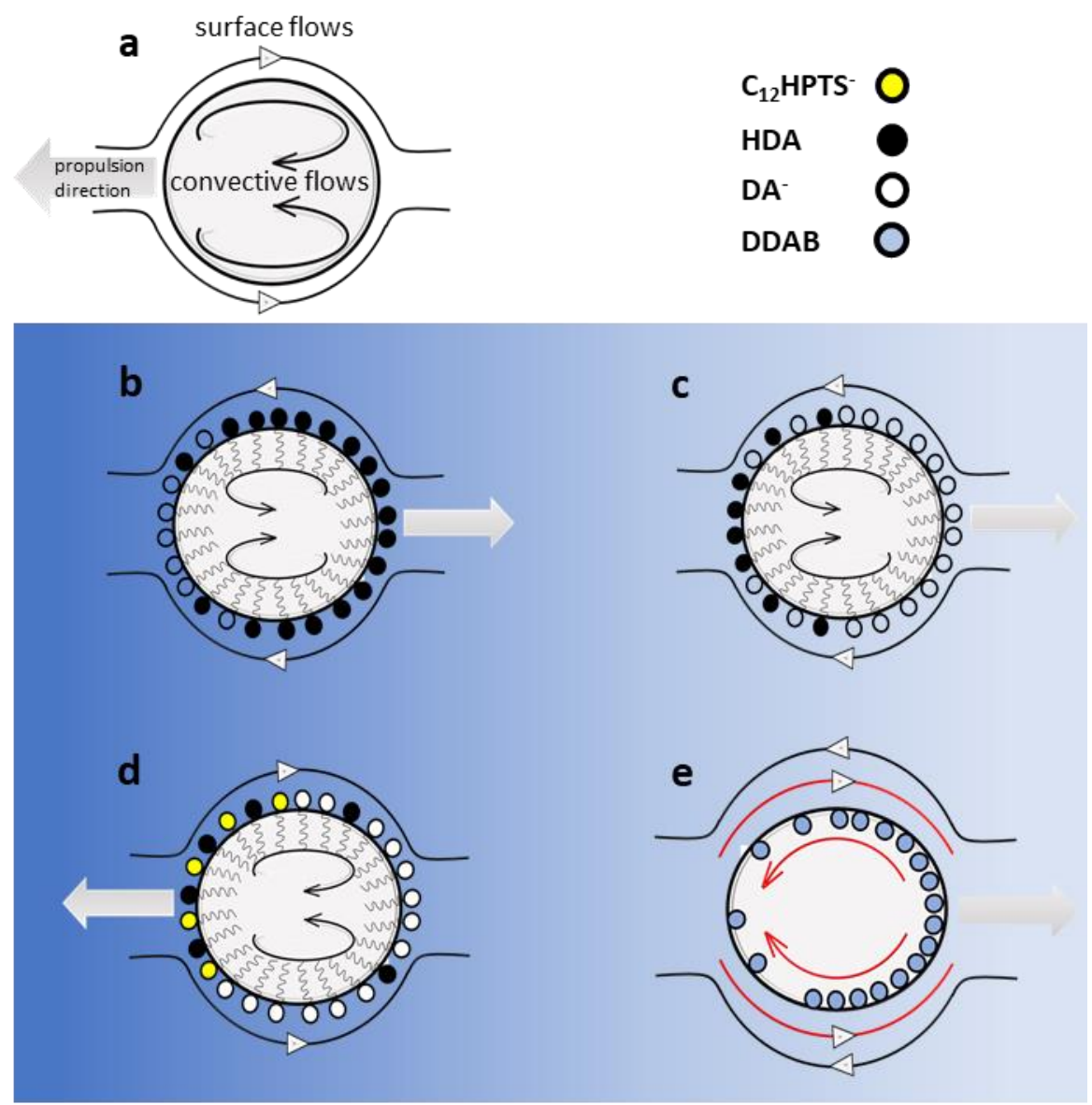

\section{Light intensity gradient}

Figure 7. Self-propulsion of droplets by the Marangoni effect. (a) A general scheme of the Marangoni effect, composed of tangential flows at the droplet surface coupled with internal circulating flows. (b) - (e) Schematic representations of the specific flows taking place in each of our systems: (b) Oil droplet in a photobase solution, (c) Oil droplet in a photoacid solution, (d) Oil droplet with a tethered photoacid, (e) Water/oil/water system containing an inner photoacid phase.

\section{Conclusions}

Here, we have developed a conceptually new method to induce self-propulsion of droplets by light. Our method is based on the use of photoacids and photobases. We present five different 
systems containing an oil droplet and a photoacid or a photobase inserted into the aqueous solution or onto the droplet surface. In the first four systems, we used dispersed light to create a non-uniform light intensity in the irradiated area. This asymmetric irradiation caused the droplet's self-propulsion. In the systems containing $6 \mathrm{MQ}$ and 2N6S in the solution, the direction of selfpropulsion was away from the light, while also exhibiting a similar trend in the change in the ST upon light-irradiation. In a system containing $\mathrm{C}_{12}$-HPTS on the surface of the droplet, we observed movement toward the light, and the change in ST upon light irradiation was also in the opposite direction as compared to the previous systems. Nevertheless, the common denominator for all these systems is the ultra-rapid change in the measured ST upon light irradiation and turning off the light on the millisecond time scales. Moreover, the change in measured ST is highly reversible, which we attribute to the fast recombination and dissociation of the photoacids and the photobases, respectively, when in their excited electronic state. This result is significant comparing to a previous study on ST kinetics in droplet systems. ${ }^{36}$ In our next system, we showed an example of compartmentalization by creating a complex droplet, containing an aqueous HPTS solution encapsulated inside an outer organic layer. These droplets exhibited a light-stimulated self-propulsion away from the light. For all of the above-described systems, we suggested a mechanism of motion, which is based on light-gradient induced ESPT between the photoacid or photobase and the surfactant, resulting in the formation of an asymmetric ST across the droplet. This asymmetric ST induces the formation of convective Marangoni flows. In our last system, we used a laser pen to drive the droplet along a defined trajectory in an HPTS solution and found a direct relationship between the concentration of HPTS and the average droplet velocity.

Overall, we have introduced here a range of attractive artificial droplet systems that are sensitive to external light stimuli using photoacids and photobases, which greatly expand our toolbox for making stimuli-responsive materials. The added novelty of our systems is the ultrafast stimuli response times (sub-ms) that are much faster than any reported system. Furthermore, our finding 
that droplet self-propulsion directionality can be manipulated via laser can result in important practical implications, especially in the field of cargo delivery for delivering chemical (medical) cargo to the reaction site as well as for systems made of several interacting components.

\section{Experimental section}

Light intensity map. Light intensities were calculated in accordance with Lambert's cosine law, which states that luminous intensity observed from a point light source is directly proportional to the cosine of the angle $\theta$ between the direction of the incident light and vector that is perpendicular to a surface: ${ }^{37} E_{\theta}=E \times \cos \theta$

The strongest light intensity (zero angle) was measured with a radiometer from a distance of 1.5 $\mathrm{cm}$. The radius ( $\mathrm{r}$ ) of each belt on the map was set as a constant distance from the central point on the petri dish, placed on the normal (vector) to the light source, where $\theta=0$. The radii were calculated by: $r=d \times \tan \theta$, where $\mathrm{d}$ is the distance from the LED (Figure S8).

Surface tension calculations. The interfacial tension measurements were conducted on an Attension Theta optical tensiometer (Biolin Scientific Inc.). The inverted pendant drop method was used to measure the interfacial tension between the oil droplet and the aqueous solutions (the exact composition of droplet and solution varies for each system and has been discussed previously). The inverted drop of oil was extracted from a syringe tip to its maximum stable size and then was allowed to stabilize before illumination cycles were conducted. The shape of the drop was fitted using the Young-Laplace equation to obtain the values of interfacial tension.

Oil droplet in a photobase solution. In the typical experiment droplet composition was: nitrobenzene:HDA (2-hexyldecanoic acid) 1:1 and $2.5 \mathrm{mM}$ of Oil Red dye. The droplet volume was $3 \mu \mathrm{L}$. The solution was prepared by addition of $15 \mu \mathrm{L}$ of $6 \mathrm{MQ}$ (6-methoxyquinoline) $1 \mathrm{M}$ in $\mathrm{MeOH}$ to $30 \mathrm{~mL}$ DI water. The final 6MQ concentration was $0.5 \mathrm{mM}$. Droplet self-propulsion was recorded with a digital camera. The video was processed with Tracker software to obtain 
droplet trajectory along with the velocity. ST was measured with optical tensiometer Theta lite. We used a hook-type needle with $d=1.052 \mathrm{~mm}$. During the typical ST measurement, we performed light on/off cycles using the same $340 \mathrm{~nm}$ LED as in the motion tracking experiment.

Oil droplet in a photoacid solution. In the typical experiment droplet composition was: nitrobenzene:HDA (2-hexyldecanoic acid) 1:1 and $2.5 \mathrm{mM}$ of Oil Red dye. The droplet volume was $3 \mu \mathrm{L}$. The aqueous solution contained $0.5 \mathrm{mM}$ of $2 \mathrm{~N} 6 \mathrm{~S}$ (2 naphthol-6-sulfonate). Droplet selfpropulsion and ST measurements were performed by the same method as in the photobase system.

Oil droplet with a tethered photoacid. In the typical experiment droplet composition was: $\mathrm{C}_{12} \mathrm{HPTS}$ 10mM, HDA 100mM, DDAB (didodecyldimethylammonium bromide) $20 \mathrm{mM}$ and Oil Red dye $2.5 \mathrm{mM}$. All the ingredients were dissolved in nitrobenzene. The droplet volume was $3 \mu \mathrm{L}$. Solution contained only DI water with $\mathrm{pH}$ 7.3. Droplet self-propulsion was recorded and processed by the same method as in the previous systems. For the ST measurements, we used a straight needle with $\mathrm{d}=1.0 \mathrm{~mm}$. During the typical ST measurement, we performed light on/off cycles using the same $405 \mathrm{~nm}$ LED as in the motion tracking experiment.

Water/oil/water system containing an inner photoacid phase. In the typical experiment outer layer of the droplet was composed of DDAB $20 \mathrm{mM}$ and Oil Red dye $2.5 \mathrm{mM}$, dissolved in nitrobenzene, and the inner layer was composed of an aqueous $10 \mathrm{mM}$ HPTS solution. The solution was composed of $50 \mathrm{mM}$ citrate buffer in $\mathrm{pH}$ 5. To begin the experiment, $5 \mu \mathrm{L}$ of the nitrobenzene solution was dripped into the petri dish making an oil droplet. Then $2 \mu \mathrm{L}$ of HPTS solution were injected inside it. Droplet self-propulsion was recorded and processed by the same method as in the previous systems. During the ST measurements, we inserted an aqueous HPTS droplet into nitrobenzene solution using a hook-type needle with $\mathrm{d}=0.712 \mathrm{~mm}$. During the typical ST measurement, we performed light on/off cycles using the same $405 \mathrm{~nm}$ LED as in the motion tracking experiment. 
Laser guided oil droplet in a photoacid solution. The droplet composition and volume were as in the first two systems, containing photobase or photoacid in the solution. The solution contained either $0.05,0.10$ or $0.20 \mathrm{mM}$ HPTS in $\mathrm{pH}$ 8. Droplet self-propulsion was recorded and processed by the same method as in the previous systems.

C12HPTS synthesis. We have followed a previously reported method to synthesize $\mathrm{C}_{12} \mathrm{HPTS}^{32}$ The synthesis contained 3 main steps. During the first step $0.7 \mathrm{mg}$ of 8-Hydroxypyrene-1,3,6Trisulfonic Acid, Trisodium Salt (HPTS) and $11 \mathrm{mg}$ of sodium acetate were added to $7 \mathrm{~mL}$ of acetic anhydride. The reaction mixture was transferred to the microwave vessel, and the reaction took place in the microwave for 1.5 hours at $130^{\circ} \mathrm{C}$. Then, the reaction mixture was filtrated with vacuum upon washing with acetone and dried in a high vacuum for $20 \mathrm{~min}$ to obtain a grey powder. During the second step, $5 \mathrm{~mL}$ of thionyl chloride was added, followed by the addition of 2 drops of DMF. Upon the addition of thionyl chloride, gas was released, and the color changed to yellowish. The vial was placed in an oil bath at $55^{\circ} \mathrm{C}$ overnight. Then the reaction mixture was poured into a $100 \mathrm{~mL}$ beaker with ice and water. Orange precipitant that formed, was collected and washed with water upon vacuum filtration. Finally, the product was dried under a high vacuum. During the third step, $1.087 \mathrm{~g}$ of DDA (Dodecylamine), $0.78 \mathrm{~mL}$ of triethanolamine and $40 \mathrm{~mL}$ DCM were added to $0.651 \mathrm{~g}$ of $\mathrm{HPTS}-\mathrm{SO}_{2} \mathrm{Cl}$, obtained in the previous step. The detailed procedure was as follows: we divided DCM into 2 flasks. The first flask was transferred to the ice bath, while the second was kept at room temperature. DDA and dodecylamine were added to the first flask, and HPTS-SO ${ }_{2} \mathrm{Cl}$ was added to the second flask. Finally, the content of the second flask (HPTS-SO ${ }_{2} \mathrm{Cl}$ solution) was added to the first flask dropwise. The color immediately changed to purple, and later to red. The mixture was left on the stirrer at room temperature for 24$48 \mathrm{~h}$. Then the resulting product was diluted with $\mathrm{NaOH} 1 \mathrm{M}$ and washed with DCM 3 times in a separating funnel. The aqueous phase, having a red color, was collected. After that, the red aqueous solution was titrated with HCL 3M until its color changed to yellow. The desired product $\mathrm{C}_{12} \mathrm{HPTS}$ was extracted from this mixture with EtOAc in a separating funnel 3 times. The organic 
phase was collected and washed with brine 1 time in a separating funnel. Finally, it was dried with $\mathrm{MgSO}_{4}$ and filtrated by filter paper. The filtrate was rotovaped. Purification was done by Chromatographic column, using hexane/EtOAc solvent mixture. The product was confirmed by TLC and mass spectroscopy.

\section{References}

(1) Zhang, X.; Chen, L.; Lim, K. H.; Gonuguntla, S.; Lim, K. W.; Pranantyo, D.; Yong, W. P.; Yam, W. J. T.; Low, Z.; Teo, W. J.; Nien, H. P.; Loh, Q. W.; Soh, S., The Pathway to Intelligence: Using Stimuli-Responsive Materials as Building Blocks for Constructing Smart and Functional Systems. Adv. Mater. 2019, 31 (11), 1804540.

(2) Merindol, R.; Walther, A., Materials learning from life: concepts for active, adaptive and autonomous molecular systems. Chem Soc Rev 2017, 46 (18), 5588-5619.

(3) Baroncini, M.; Silvi, S.; Credi, A., Photo- and Redox-Driven Artificial Molecular Motors. Chem Rev 2020, 120 (1), 200-268.

(4) Grzybowski, B. A.; Huck, W. T., The nanotechnology of life-inspired systems. Nat Nanotechnol 2016, 11 (7), 585-92.

(5) Kageyama, Y., Light-Powered Self-Sustainable Macroscopic Motion for the Active Locomotion of Materials. ChemPhotoChem 2019, 3 (6), 327-336.

(6) Lach, S.; Yoon, S. M.; Grzybowski, B. A., Tactic, reactive, and functional droplets outside of equilibrium. Chem. Soc. Rev 2016, 45 (17), 4766-4796.

(7) Hanczyc, M. M.; Toyota, T.; Ikegami, T.; Packard, N.; Sugawara, T., Fatty Acid Chemistry at the Oil-Water Interface: Self-Propelled Oil Droplets. J. Am. Chem. Soc. 2007, 129 (30), 9386-9391.

(8) Toyota, T.; Maru, N.; Hanczyc, M. M.; Ikegami, T.; Sugawara, T., Self-Propelled Oil Droplets Consuming “Fuel” Surfactant. J. Am. Chem. Soc. 2009, 131 (14), 5012-5013.

(9) Tanabe, T.; Ogasawara, T.; Suematsu, N. J., Effect of a product on spontaneous droplet motion driven by a chemical reaction of surfactant. Phys. Rev. E 2020, 102 (2), 023102.

(10) Jamaluddin, S. J. S.; Khaothong, K.; Tinsley, M. R.; Showalter, K., Photochemical motion control of surface active Belousov-Zhabotinsky droplets. Chaos: An Interdisciplinary Journal of Nonlinear Science 2020, 30 (8), 083143.

(11) Suematsu, N. J.; Saikusa, K.; Nagata, T.; Izumi, S., Interfacial Dynamics in the Spontaneous Motion of an Aqueous Droplet. Langmuir 2019, 35 (35), 11601-11607.

(12) Jin, C.; Krüger, C.; Maass, C. C., Chemotaxis and autochemotaxis of self-propelling droplet swimmers. Proc. Natl. Acad. Sci. U.S.A. 2017, 114 (20), 5089.

(13) Izri, Z.; van der Linden, M. N.; Michelin, S.; Dauchot, O., Self-Propulsion of Pure Water Droplets by Spontaneous Marangoni-Stress-Driven Motion. Physical Review Letters 2014, 113 (24), 248302.

(14) Matsushita, S.; Tanaka, S.; Yoshida, K.; Kobayashi, K.; Tsuruki, Y.; Shibuya, Y.; Isobe, T.; Nakajima, A., Activation of the spontaneous motion of a nitrobenzene droplet by chlorobenzene blending. Colloids Surf., A 2012, 395, 233-239.

(15) Banno, T.; Kuroha, R.; Toyota, T., pH-Sensitive Self-Propelled Motion of Oil Droplets in the Presence of Cationic Surfactants Containing Hydrolyzable Ester Linkages. Langmuir 2012, 28 (2), 1190-1195. 
(16) Nagai, K.; Sumino, Y.; Yoshikawa, K., Regular self-motion of a liquid droplet powered by the chemical marangoni effect. Colloids Surf., B 2007, 56 (1), 197-200.

(17) Francis, W.; Fay, C.; Florea, L.; Diamond, D., Self-propelled chemotactic ionic liquid droplets. Chem. Commun. 2015, 51 (12), 2342-2344.

(18) Schmitt, M.; Stark, H., Marangoni flow at droplet interfaces: Three-dimensional solution and applications. Physics of Fluids 2016, 28 (1), 012106.

(19) Yoshinaga, N.; Nagai, K. H.; Sumino, Y.; Kitahata, H., Drift instability in the motion of a fluid droplet with a chemically reactive surface driven by Marangoni flow. Phys. Rev. E 2012, 86 (1), 016108.

(20) Ban, T.; Yamagami, T.; Nakata, H.; Okano, Y., pH-Dependent Motion of Self-Propelled Droplets due to Marangoni Effect at Neutral pH. Langmuir 2013, 29 (8), 2554-2561.

(21) Lagzi, I.; Soh, S.; Wesson, P. J.; Browne, K. P.; Grzybowski, B. A., Maze Solving by Chemotactic Droplets. J. Am. Chem. Soc. 2010, 132 (4), 1198-9.

(22) Miura, S.; Banno, T.; Tonooka, T.; Osaki, T.; Takeuchi, S.; Toyota, T., pH-Induced Motion Control of Self-Propelled Oil Droplets Using a Hydrolyzable Gemini Cationic Surfactant. Langmuir 2014, 30 (27), 7977-7985.

(23) Ichimura, K.; Oh, S.-K.; Nakagawa, M., Light-driven motion of liquids on a photoresponsive surface. Science 2000, 288 (5471), 1624-1626.

(24) Florea, L.; Wagner, K.; Wagner, P.; Wallace, G. G.; Benito-Lopez, F.; Officer, D. L.; Diamond, D., Photo-Chemopropulsion - Light-Stimulated Movement of Microdroplets. Adv. Mater. 2014, 26 (43), 7339-7345.

(25) Zarghami, S.; Xiao, Y.; Wagner, P.; Florea, L.; Diamond, D.; Officer, D. L.; Wagner, K., Dual Droplet Functionality: Phototaxis and Photopolymerization. ACS Appl. Mater. Interfaces 2019, 11 (34), 31484-31489.

(26) Xiao, Y.; Zarghami, S.; Wagner, K.; Wagner, P.; Gordon, K. C.; Florea, L.; Diamond, D.; Officer, D. L., Moving Droplets in 3D Using Light. Adv. Mater. 2018, 30 (35), 1801821.

(27) Pines, D.; Pines, E., Solvent Assisted Photoacidity. In Hydrogen-Transfer Reactions, 2006; pp 377-415.

(28) Arnaut, L. G.; Formosinho, S. J., Excited-state proton transfer reactions I. Fundamentals and intermolecular reactions. J. Photochem. Photobiol., A 1993, 75 (1), 1-20.

(29) Lagzi, I., Self-division of a mineral oil-fatty acid droplet. Chem. Phys. Lett. 2015, 640, 14.

(30) Derenyi, I.; Lagzi, I., Fatty acid droplet self-division driven by a chemical reaction. Phys. Chem. Chem. Phys. 2014, 16 (10), 4639-4641.

(31) Amdursky, N.; Rashid, M. H.; Stevens, M. M.; Yarovsky, I., Exploring the binding sites and proton diffusion on insulin amyloid fibril surfaces by naphthol-based photoacid fluorescence and molecular simulations. Sci. Rep. 2017, 7 (1), 6245.

(32) Amdursky, N.; Lin, Y.; Aho, N.; Groenhof, G., Exploring fast proton transfer events associated with lateral proton diffusion on the surface of membranes. Proc Natl Acad Sci U S A 2019, 116 (7), 2443-2451.

(33) Yucknovsky, A.; Mondal, S.; Burnstine-Townley, A.; Foqara, M.; Amdursky, N., Use of Photoacids and Photobases To Control Dynamic Self-Assembly of Gold Nanoparticles in Aqueous and Nonaqueous Solutions. Nano Letters 2019, 19 (6), 3804-3810.

(34) Rybalko, S.; Magome, N.; Yoshikawa, K., Forward and backward laser-guided motion of an oil droplet. Phys Rev E Stat Nonlin Soft Matter Phys 2004, 70 (4 Pt 2), 046301.

(35) Maass, C. C.; Krüger, C.; Herminghaus, S.; Bahr, C., Swimming Droplets. Annu. Rev. Condens. Matter Phys. 2016, 7 (1), 171-193.

(36) Haimov, B.; Iakovlev, A.; Glick-Carmi, R.; Bloch, L.; Rich, B. B.; Müller, M.; Pokroy, B., Kinetics of Nanoscale Self-Assembly Measured on Liquid Drops by Macroscopic Optical Tensiometry: From Mercury to Water and Fluorocarbons. J Am Chem Soc 2016, 138 (8), 258591. 


\section{Acknowledgments}

A.Y. thanks the Ariane de Rothschild fellowship for financial support. N.A. thanks the Binational Science Foundation (number 2018239), and the Ministry of Science and Technology (number 316243), for financial support. We thank the Russel Berrie Nanotechnology Institute (RBNI) and the Grand Technion Energy Program (GTEP) for their support in equipment use.

\section{Author contributions}

A.Y. and N.A. designed the project. A.Y. B.B.R and A.W. performed the experiment. A.Y. and N.A. wrote the first draft of the manuscript. B.B.R. and B.P. co-wrote the manuscript. N.A. and B.P. supervised the research.

\section{Data availability}

The data that support the findings of this study are available within the paper as well as in the Supplementary Information.

\section{Notes}

The authors declare no competing interests. 